\title{
Erratum: An Approximation Algorithm for Minimum-Cost Vertex-Connectivity Problems
}

\author{
R. Ravi* \\ GSIA, Carnegie Mellon University
}

\author{
David P. Williamson ${ }^{\dagger}$ \\ IBM Almaden
}

July 2001

\begin{abstract}
There is an error in our paper "An Approximation Algorithm for Minimum-Cost VertexConnectivity Problems," (Algorithmica (1997) 18:21-43). In that paper we consider the following problem: given an undirected graph and values $r_{i j}$ for each pair of vertices $i$ and $j$, find a minimum-cost set of edges such that there are $r_{i j}$ vertex-disjoint paths between vertices $i$ and $j$. We gave approximation algorithms for two special cases of this problem. Our algorithms rely on a primal-dual approach which has led to approximation algorithms for many edge-connectivity problems. The algorithms work in a series of stages; in each stage an augmentation subroutine augments the connectivity of the current solution. The error is in a lemma for the proof of the performance guarantee of the augmentation subroutine.

In the case $r_{i j}=k$ for all $i, j$, we described a polynomial-time algorithm that claimed to output a solution of cost no more than $2 \mathcal{H}(k)$ times optimal, where $\mathcal{H}(n)=1+\frac{1}{2}+\cdots+\frac{1}{n}$. This result is erroneous. We describe an example where our primal-dual augmentation subroutine, when augmenting $k$-vertex connected graph to a $k+1$-vertex connected graph, gives solutions that are a factor $\Omega(k)$ away from the minimum.

In the case $r_{i j} \in\{0,1,2\}$ for all $i, j$, we gave a polynomial-time algorithm which outputs a solution of cost no more than 3 times the optimal. In this case, we prove that the erroneous statement in the lemma does not apply, and that the algorithm performs as claimed.
\end{abstract}

\section{Background}

Let $G=(V, E)$ be an undirected graph with non-negative $\operatorname{costs} c_{e} \geq 0$ on all edges $e \in E$. In the survivable network design problem, one is given a non-negative integer $r_{i j}$ for each pair of vertices $i, j$, and must find a minimum-cost set of edges $E^{\prime} \subseteq E$ such that for every $i, j$ pair there are at least $r_{i j}$ vertex-disjoint paths between $i$ and $j$ in the graph $\left(V, E^{\prime}\right)$. The minimum-cost $k$-vertex-connectivity problem is a special case of the survivable network design problem in which $r_{i j}=k$ for all pairs of vertices $i, j$.

In Ravi and Williamson [10], we gave polynomial-time approximation algorithms for the minimum-cost $k$-vertex connectivity problem, and the survivable network design problem when $r_{i j} \in\{0,1,2\}$ for all $i, j$. We abbreviate the former problem as $k$-VC and the latter as $\{0,1,2\}$ SNDP. We claimed that the algorithms returned solutions of cost within $2 \mathcal{H}(k)$ of optimal, and 3 times optimal, respectively, where $\mathcal{H}(n)=1+\frac{1}{2}+\cdots+\frac{1}{n}$. The first claim is erroneous, and

*Address: GSIA, Carnegie Mellon University, Pittsburgh, PA 15213-3890. Email: ravi@cmu.edu.

†Address: IBM Almaden Research Center, 650 Harry Rd. K53/B1, San Jose, CA 95120. Email: dpw@almaden.ibm. com. 
in this note we describe the error and give an example showing that our algorithm can be quite far from optimal for $k$-VC. However, our 3 -approximation algorithm for $\{0,1,2\}$-SNDP is still correct, and in this note we show why the error that causes problems in the previous case does not affect this algorithm.

In what follows, we assume that the reader is familiar with the notation, algorithms, and proofs of [10]. Our paper flowed out of a line of research on designing approximation algorithms for edge-connectivity problems $[1,5,6,8,11]$. The algorithms of our paper followed the general approach of these algorithms. The algorithms break down the problem into a number of phases. In each phase, we specify certain vertex sets $S, S^{\prime}$ that must be augmented; that is, we must select an additional edge from $\delta\left(S: S^{\prime}\right)$ for each specified $S, S^{\prime}$, where $\delta\left(S: S^{\prime}\right)$ is the set of edges with one endpoint in $S$ and one in $S^{\prime}$. This augmentation problem is formulated as an integer programming problem, and the problem is solved by using a variant of the primal-dual method.

The error in [10] is contained in the proof of the performance guarantee of the primal-dual algorithm for the augmentation problem. Specifically, there is an erroneous statement in Lemma 4.7. We describe the error in Section 2 of this note. We had claimed that the performance guarantee for each phase of the algorithm would hold if three conditions held on a function $h$ encoding the vertex sets $S, S^{\prime}$ that needed to be augmented. These three conditions are summarized in page 36 (just before Corollary 4.8) in [10]. Because of the error in Lemma 4.7, Corollary 4.8 as stated is wrong. We elaborate on the consequences for the two main results in [10] below.

In the case of $k$-VC, we do not have a $2 \mathcal{H}(k)$-approximation algorithm as claimed because Lemma 4.7 does not hold when applied to the algorithm for the augmentation problems arising in phases of the $k$-vertex-connectivity algorithm. In Section 3 of this note, we describe a counterexample to the claimed performance guarantee. The example shows that the algorithm used for augmenting a $p$-vertex-connected graph to a $(p+1)$-vertex-connected graph can give a solution of cost a factor of $\Omega(p)$ more than the optimum cost. Thus Theorem 4.1 is wrong for this case, as are Theorem 4.2 and Corollary 4.3 .

However, for $\{0,1,2\}$-SNDP, the statement that is incorrect in Lemma 4.7 in general can be shown to hold. In Section 4 of this note, we provide a complete proof. This also shows that Theorem 4.1 is true for the $\{0,1,2\}$-SNDP, and consequently shows that Theorem 4.4 is correct.

\section{The Error in the Proof of Lemma 4.7}

The strategy used in [10] to prove Theorem 4.1 and Corollary 4.8 follows that in Section 5 of Williamson et al. [11]. The idea is to use induction over the iterations of the primal-dual algorithm and show that in any iteration,

$$
\sum_{C \in \mathcal{C}}\left|\delta_{F^{\prime}}\left(C: \zeta_{I}(C)\right)\right| \leq 2|\mathcal{C}|
$$

Here $\mathcal{C}$ is the collection of active sets in this iteration, $F^{\prime}$ is a minimal set of edges needed to solve the augmentation problem, and $I$ is a set of preexisting edges from previous phases. A proof of this inequality in every iteration implies the performance guarantee given in Theorem 4.1.

Consider the set of edges $Y$ defined as $Y=\bigcup_{C \in \mathcal{C}} \delta_{F^{\prime}}(C: \zeta(C))$. These edges must have been added during or after the current iteration. We show in Lemma 4.5 the existence of a witness set for each edge. For each edge $e \in Y$ there exists a witness set $S_{e} \subset V$ such that

1. $\delta_{F^{\prime}}\left(S_{e}: \zeta\left(S_{e}\right)\right)=\{e\}$,

2. $S_{e}$ is violated in the current iteration, 


\section{For each $C \in \mathcal{C}$ either $C \subseteq S_{e}$ or $C \cap S_{e}=\emptyset$.}

This lemma continues to hold. We then considered a collection $\mathcal{S}$ of sets $S_{e}$ satisfying the conditions of Lemma 4.5, taken over all the edges $e$ in $Y$. We called $\mathcal{S}$ a witness family and required that there exists a laminar witness family for the function $h$ defined in the augmentation problem. Lemmas 4.12 and 4.13 are also correct; these lemmas show the existence of laminar witness families for the augmentation problems arising from the $k$-VC and $\{0,1,2\}$-SNDP.

For the remaining proof of the inequality, we essentially followed the proof of Williamson et al. in [11]. We first added the set $V$ to the witness family. The family can then be viewed as defining a tree $H$ with a vertex $v_{S}$ for each $S \in \mathcal{S}$ and edge $\left(v_{S}, v_{T}\right)$ if $T$ is the smallest element of $\mathcal{S}$ properly containing $S$. To each active set $C \in \mathcal{C}$ we associated the smallest set $S \in \mathcal{S}$ that contains it. We colored the vertices of the tree $H$ : a vertex $v_{S}$ is colored red if $S$ is associated with some active set $C$ and colored blue otherwise. Let $\mathcal{L}\left(v_{S}\right)$ be the collection of active sets associated with a red vertex $v_{S}$.

In Lemma 4.6, we showed the simple fact that the tree $H$ has at most one blue leaf.

In Lemma 4.7, we claimed that for any red vertex $v_{S}$ in $H$, the degree of $v_{S}$ in the tree $H$ is at least $\sum_{C \in \mathcal{L}\left(v_{S}\right)}\left|\delta_{F^{\prime}}(C: \zeta(C))\right|$. The lemma is used in a charging scheme that implies inequality (1). This lemma is wrong. The proof went as follows:

Note that the one-to-one mapping between the edges of $Y$ and the witness sets implies a one-to-one mapping between the edges of $Y$ and the edges of $H$ : each witness set $S$ defines a unique edge $\left(v_{S}, v_{T}\right)$ of $H$, where $T$ contains $S$. Consider any edge $e \in \delta_{F^{\prime}}(C: \zeta(C))$ for some $C \in \mathcal{C}$. Let $\left(v_{S_{e}}, v_{T}\right)$ be the edge defined by the witness set $S_{e}$. The active set $C$ must be associated with either $v_{S_{e}}$ or $v_{T}$.

The error in the proof is in this last statement. Since $C$ is a minimal violated set and $S_{e}$ is a violated set, we know that either $C \subseteq S_{e}$ or $C \cap S_{e}=\emptyset$. We prove below (in Claim 4.1) that if $C \subseteq S_{e}$ then $C$ is associated with $S_{e}$. However, it is not in general the case that if $C \cap S_{e}=\emptyset$ then $C$ is associated with $T$.

In the case of edge connectivity, the proofs in Williamson et al. [11] consider edges $Y=$ $\bigcup_{C \in \mathcal{C}} \delta_{F^{\prime}}(C)$, and $e \in Y$. It is not hard to see that the problematic statement of Lemma 4.7 is true in this case. Suppose not. If $C \cap S_{e}=\emptyset$, then $C$ is associated with some witness set $X$, and either $X \supset T \supset S_{e}$ or $X \cap S_{e}=\emptyset$. In the former case, $e$ has one endpoint in $S_{e} \subset T$ and one in $C \subseteq X-T$. Thus $e \in \delta_{F^{\prime}}(T)$, which either contradicts property (1) of the witness set $T$, or the property that there is exactly one set in $\mathcal{S}$ that is a witness set for $e$. In the latter case, $e$ has an endpoint in $S_{e} \subset V-X$ and another in $C \subseteq X$, so that $e \in \delta_{F^{\prime}}(X)$. This leads to a contradiction as above.

However, as we will show in the next section, this proof breaks down in the case of vertex connectivity, since it can be the case that $e \in \delta_{F^{\prime}}(T)$ but $e \notin \delta_{F^{\prime}}(T: \zeta(T))$. Thus this does not contradict the property of $T$ as a witness set or $\mathcal{S}$ as a witness family.

This renders Lemma 4.7, and consequently Theorem 4.1 and Corollary 4.8 incorrect. In the next section, we show a counterexample to these three claims for the $k$-vertex-connectivity augmentation problem. In the following section, however, we "salvage" our result for the $\{0,1,2\}$ SNDP in [10] and show why the counterparts of these claims (the part of Theorem 4.1 for $\{0,1,2\}$-SNDP, and consequently Theorem 4.4) continue to hold. 


\section{The Error in the Algorithm for $k$-VC}

We recall the high-level structure of the algorithm for $k$-VC. The algorithm consists of $k$ phases, and each phase adds edges to the current solution, starting initially with the empty set of edges at the beginning of phase 1 . The idea is that at the end of phase $p$, our set of edges should form a $p$-vertex-connected graph on the set of vertices of the input graph. We denote our set of edges at the end of phase $p$ as $F_{p}$. In phase $p$ we must augment the $(p-1)$-vertex-connected edge set $F_{p-1}$ to the $p$-vertex-connected $F_{p}$.

Figure 1 shows an example where the inequality (1) fails to hold, in the case of augmenting from a $k$-vertex connected graph to a $(k+1)$-vertex connected graph. The example is structured as follows. The dashed edges are those added in the augmentation; all other edges are present from previous phases. Let $F^{\prime}$ be the set of augmentation edges and $I$ be the set of all other edges. Each rectangle with a number $x$ in it denotes a complete graph on $x$ vertices. Pairs of rectangles connected by two lines means that there is a complete bipartite graph between the nodes of the two cliques. Similarly, the nodes $v_{i}$ connected to the rectangles labeled $k$ at the bottom of the figure have edges to all $k$ vertices in those cliques. (The thick and dashed edges incident to the

$w_{j}$ nodes are single edges). We define some sets of nodes. Let set $S_{i, j}$ be the set of vertices containing $v_{i}$ and the first $j$ cliques above it (that is, those labeled $k, k-1, \ldots, k-j+1$ ). By definition, $S_{i, 0}=\left\{v_{i}\right\}$.

In the case of this example, the active sets $\mathcal{C}$ consist of the singleton sets of the vertices $v_{1}, \ldots, v_{k}$ and $w_{1}, \ldots, w_{k / 2}$, since each of these has exactly $k$ node neighbors. In the augmentation, edges are added from $w_{1}$ to each of $v_{1}, v_{2}, \ldots, v_{k}, w_{2}$ to some node in each of the $k$-cliques, $w_{3}$ to each of the $(k-1)$-cliques, and so on. We claim that this is a minimal augmentation. In particular, we claim that the sets $S_{i, j}$ are witness sets for the augmentation edges for $i=1, \ldots, k$, $j=0, \ldots, k / 2+2$. Observe that each of these sets has only $k$ node neighbors without the augmentation edges, and that the augmenting edges add exactly one node neighbor (one of the $w_{i}$ ) to each of these sets.

Hence for this example we have that $\left|\delta_{F^{\prime}}\left(v_{i}: \zeta_{I}\left(v_{i}\right)\right)\right|=1,\left|\delta_{F^{\prime}}\left(w_{i}: \zeta_{I}\left(w_{i}\right)\right)\right|=k$, and $|\mathcal{C}|=3 k / 2$, yielding that

$$
\sum_{C \in \mathcal{C}}\left|\delta_{F^{\prime}}\left(C: \zeta_{i}(C)\right)\right|=k+k^{2} / 2 \not \leq 3 k=2|\mathcal{C}|,
$$

contradicting inequality (1) for $k>4$.

By adding appropriate edge costs, and modifying the example slightly, one can give an example in which the optimal augmentation costs are $\frac{7}{2} k$, and the primal-dual algorithm finds an augmentation of cost $k+k(k-1) / 2$.

Even in the case of augmenting a 2-vertex connected graph to be 3 -vertex connected, we can construct examples where the number of active sets is $p$ while the "active degree" (the left-handside term in the inequality above) is $3(p-2)$ for any $p \geq 4$. This shows that our augmentation algorithm does not provide the claimed 2-approximation guarantee even in this case. However, we show in the next section that our claimed guarantee holds for smaller values of vertex connectivity, i.e., for the $\{0,1,2\}$-Survivable Network Design Problem.

\section{Corrected Proof for the $\{0,1,2\}$-Survivable Network Design Problem}

In this section we show that Lemma 4.7 holds in the case of $\{0,1,2\}$-SNDP. 


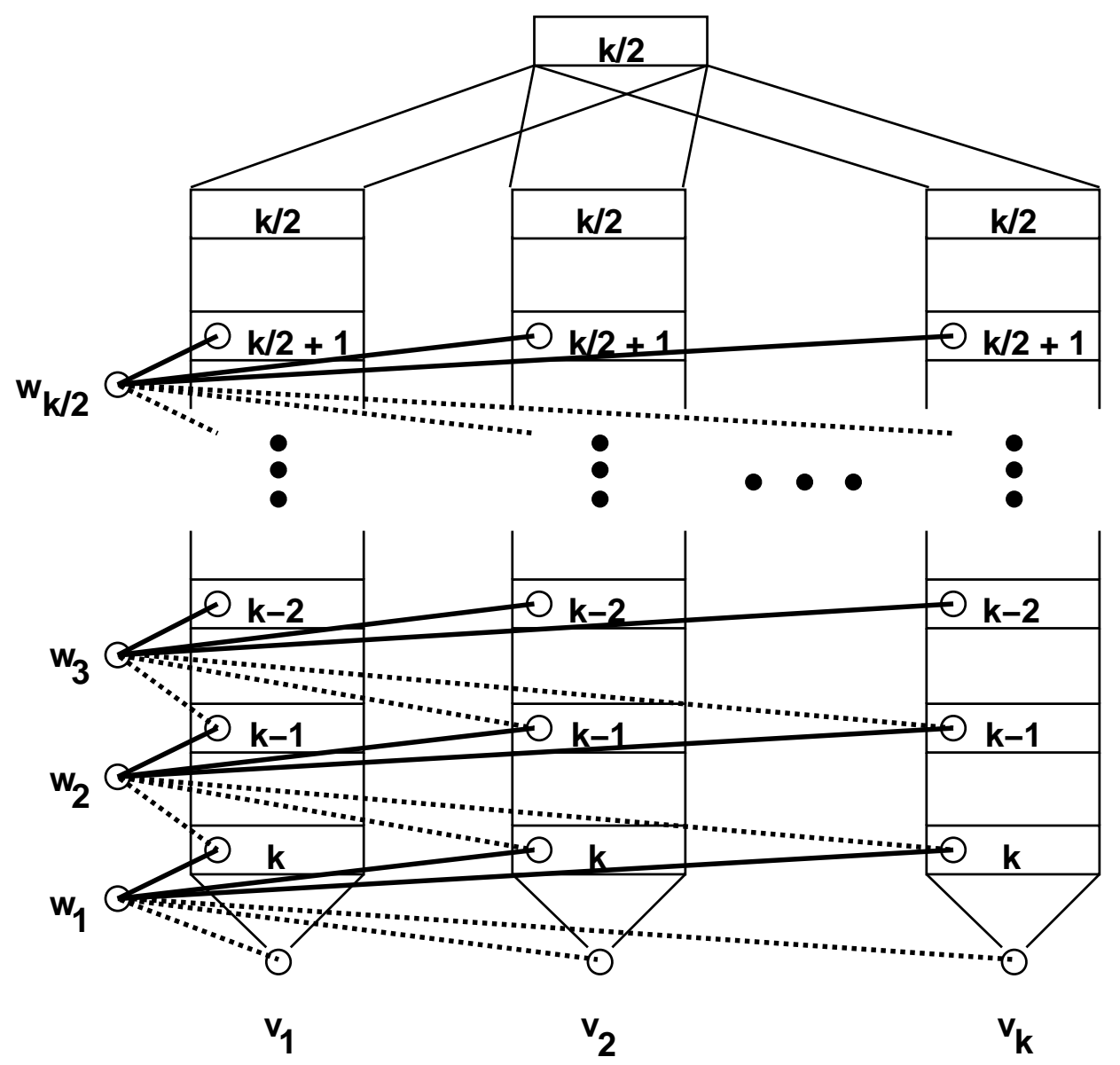

Figure 1: Bad example for inequality (1) for the $k$-VC problem. 
First, we recall the high-level algorithm for solving the survivable network design problem when $r_{i j} \in\{0,1,2\}$. The algorithm here will have two phases. In the first phase, we find a solution for the network design problem with requirements $r_{i j}^{\prime}=\max \left(r_{i j}-1,0\right)$. In the second phase, we augment this solution to find a feasible solution for the original problem. Essentially the first phase finds a set of edges to connect all pairs of vertices $i, j$ for which $r_{i j}=2$, and the second phase finds a second vertex-disjoint path for these pairs of vertices, as well as edges to connect all pairs $i, j$ for which $r_{i j}=1$.

Notice that when finding a set of edges to solve the problem of the first phase, there is no difference between edge-connectivity and vertex-connectivity. Hence we can use an approximation algorithm for the edge-connectivity survivable network design problem in which $r_{i j}^{\prime} \in\{0,1\}$. The first such algorithm for this problem is due to Agrawal, Klein, and Ravi [1]. For the sake of our analysis, we use the algorithm of Goemans and Williamson [5]. Let $F_{1}$ denote the set of edges returned by this algorithm.

To find an augmenting set of edges in the second phase, we once again use the augmenting subroutine Augment. We call Augment on a modified graph $G^{\prime}=\left(V^{\prime}, E^{\prime}\right)$. For every edge $(i, j) \in F_{1}$ such that $r_{i j}=2$, we create a new vertex $u$ that subdivides the edge $(i, j)$. That is, for each such edge $(i, j)$, we add $u$ to $V$, remove $(i, j)$ from $E$, and add edges $(i, u)$ and $(u, j)$. We split the cost of the edge $(i, j)$ equally between the two new edges; i.e., we set $c_{i u}=c_{u j}=\frac{c_{i j}}{2}$. Our reason for doing this subdivision is so that $i$ and $j$ are no longer adjacent in the modified graph, and so that we will be able to apply Menger's Theorem. Note that there is a solution $F_{1}^{\prime}$ in $G^{\prime}$ corresponding to $F_{1}$ in $G$.

To use Augment, we set $h(S)=1$ exactly when there exist $i \in S, j \in \zeta_{F_{1}^{\prime}}(S)$ (in the modified graph $\left.G^{\prime}\right)$ such that $\left|\Gamma_{F_{1}^{\prime}}(S)\right|<r_{i j}$. This can happen in one of two ways. If $r_{i j}=2$, then $i$ and $j$ need to be 2-vertex-connected but are separated by a cutvertex. If $r_{i j}=1$ then $i$ and $j$ need to be connected, but are in different connected components. AUGMENT will return a set of edges $F^{\prime} \subseteq E^{\prime}-F_{1}^{\prime}$ such that $\left|\delta_{F^{\prime}}\left(S: \zeta_{F_{1}^{\prime}}(S)\right)\right| \geq 1$ whenever $h(S)=1$. By the definition of $h$ and $F_{1}^{\prime}$, for any two $i, j$ such that $r_{i j}=2$, the set of edges $F_{1}^{\prime} \cup F^{\prime}$ will contain no cutvertex separating $i$ and $j$ in the modified graph. Similarly, for any two $i, j$ such that $r_{i j}=1$, there will exist a path in $F_{1}^{\prime} \cup F^{\prime}$ from $i$ to $j$ in the modified graph. Notice that since there are no edges in $E^{\prime}-F_{1}^{\prime}$ involving the new vertices of the modified graph, the set of edges $F^{\prime}$ is a subset of edges of the original graph. Thus $F_{2} \leftarrow F_{1} \cup F^{\prime}$ is a feasible solution to the original problem in the original graph: the non-existence of a cutvertex separating any $i, j$ with $r_{i j}=2$ in the modified graph implies that there are two vertex-disjoint paths between $i$ and $j$ in the edge set $F_{2}$, and similarly that $i$ and $j$ are connected in $F_{2}$ if $r_{i j}=1$.

For the first (Edge-SNDP) phase, the performance guarantee correctly follows from Agrawal, Klein, and Ravi [1], or Goemans and Williamson [5]. For the proof of performance guarantee of the Augment procedure in the second phase, recall that in Lemma 4.7, we made the following key claim that was erroneous for the $k$-VC.

Claim 4.1 Consider an edge $e \in \delta_{F^{\prime}}(C: \zeta(C))$ for some $C \in \mathcal{C}$. Let $\left(v_{S_{e}}, v_{T}\right)$ be the edge defined by the witness set $S_{e}$. The active set $C$ must be associated with either $v_{S_{e}}$ or $v_{T}$.

We now give a detailed proof of the above claim in this case.

Proof: We consider two cases: $C \subseteq S_{e}$ or $C \cap S_{e}=\emptyset$. These are exhaustive since an active set such as $C$ cannot cross a violated witness set such as $S_{e}$.

Case 1: $C \subseteq S_{e}$.

First, note that if $S_{e}$ is a witness set for edge $e$, then $\delta_{F^{\prime}}\left(S_{e}: \zeta\left(S_{e}\right)\right)=\{e\}$. Suppose for a contradiction that $C$ is not associated with $S_{e}$ in this case. Then, there must be a $S^{\prime}$ such that 


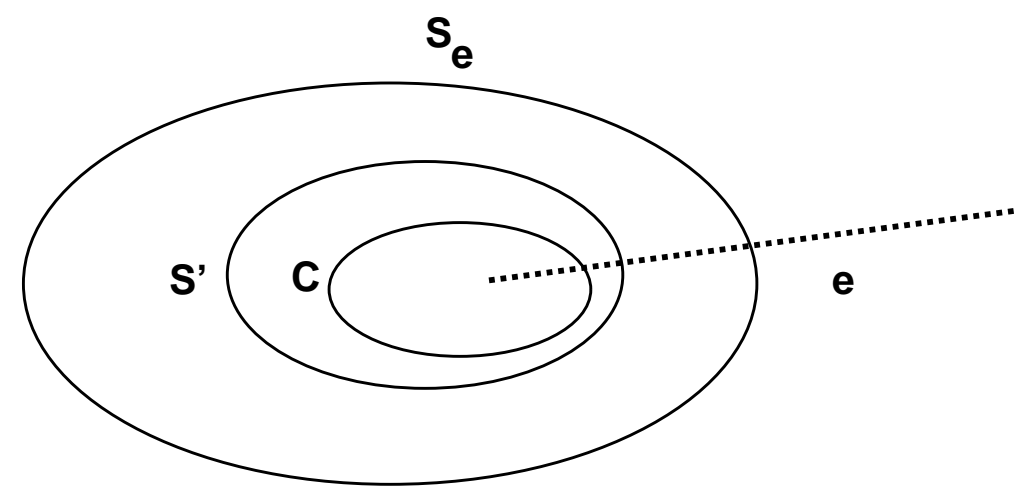

Figure 2: Case 1 of Claim 4.1.

$C \subseteq S^{\prime} \subseteq S_{e}$ and $S^{\prime}$ is in the laminar witness family. We claim this implies that $\delta_{F^{\prime}}\left(S^{\prime}: \zeta\left(S^{\prime}\right)\right) \ni e$. We know $C \subseteq S^{\prime}$ which contains one endpoint of $e$. Also, since $\Gamma\left(S^{\prime}\right) \subseteq S_{e} \cup \Gamma\left(S_{e}\right), \zeta\left(S_{e}\right) \subseteq \zeta\left(S^{\prime}\right)$. Thus the other endpoint of $e$, which is in $\zeta\left(S_{e}\right)$, must also be in $\zeta\left(S^{\prime}\right)$, and $e \in \delta_{F^{\prime}}\left(S^{\prime}: \zeta\left(S^{\prime}\right)\right.$ ). However, $S^{\prime}$ cannot be a witness set for otherwise there would be two witnesses in the family for $e$, a contradiction. See Figure 2 for an illustration of this case.

We note that the proof of this case does not use the restricted setting of the $\{0,1,2\}$-vertexconnectivity problem.

Case 2: $C \cap S_{e}=\emptyset$.

Let $e=(u, v)$ where $u \in S_{e}$ and $v \in C$. Let the parent of $S_{e}$ in the laminar witness family be $T$, and suppose for a contradiction that $C$ is not associated with $T$. This implies that $C \nsubseteq T$ or that $T \cap C=\emptyset$.

In this case we first note that $v$ must belong to $\Gamma(T)$, the node-neighborhood of $T$. For otherwise, $e$ would be in $\delta_{F^{\prime}}(T: \zeta(T))$ and there would be two sets in the family $\left(S_{e}\right.$ and $\left.T\right)$ witnessing $e$.

This implies that $T$ must be a witness set of type (2), i.e., there is a pair $i, j$ of nodes with $i \in T$ and $j \in \zeta(T)$ with $r_{i j}=2$ but $i$ and $j$ are separated by the cutvertex $v$ in the current graph $F_{1} \cup F$ (when $C$ was active; see Figure 3 ). Note that $v$ must be this cutvertex since $\Gamma_{F_{1} \cup F}=\{v\}$. By the property of phase 1 of the algorithm, there is a path already between $i$ and $j$ in the current graph and $v$ is a cutvertex separating them lying on this path.

The key observation then is that $j \in C$ since otherwise $C$, which contains $v$ on the path from $i$ to $j$, will have at least two node neighbors in the current graph along this path, one in $T$ and the other in $V-T-\{v\}$, contradicting that $C$ is active (See Figure 3).

Now, since $T$ is violated of type (2), we infer that $\zeta(T)$ is also violated (since $\Gamma(\zeta(T)) \subset \Gamma(T)$ ) and this is not empty since it contains $j$ and not $i$, which means $\Gamma(\zeta(T))=\{v\}$.

But then we see that $\zeta(T)$ and $C$ cross in this case (since $v$ is in $C$ and not in $\zeta(T)$ and also $\zeta(T) \nsubseteq \subseteq$ since $C$ is minimally violated), a contradiction.

This shows that the 3 -approximation guarantee of our two-stage algorithm correctly holds for the $\{0,1,2\}$-Vertex-Connectivity problem. 


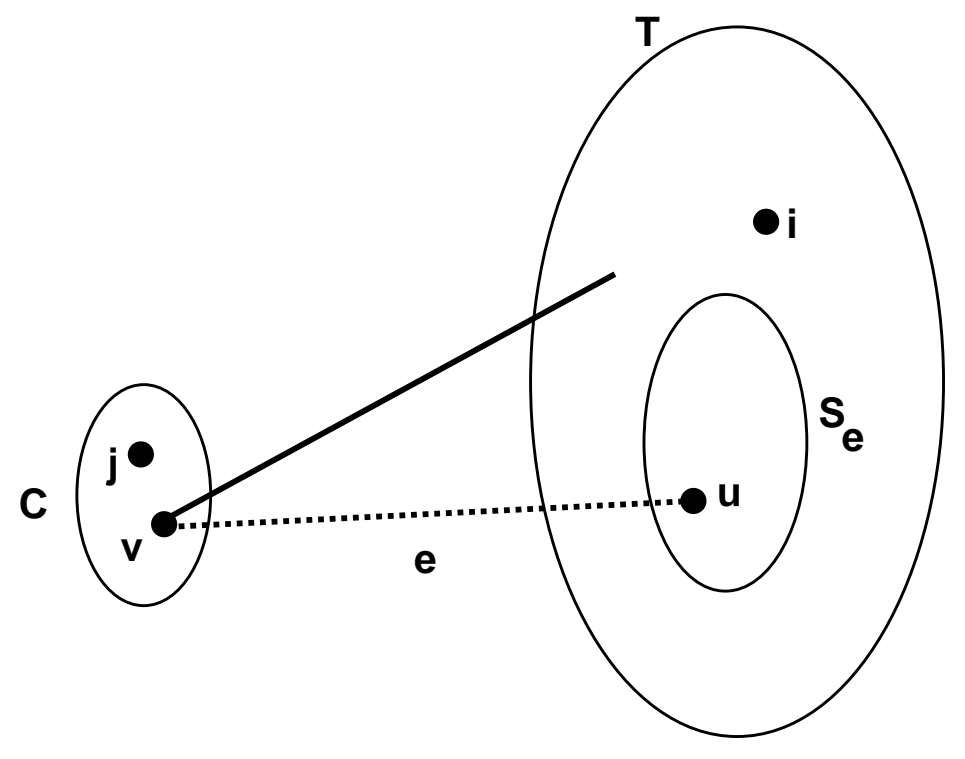

Figure 3: Case 2 of Claim 4.1.

\section{Concluding Remarks}

We remark that the structural lemmas (Lemmas 4.9-12) in our paper [10] are correct and may be useful in other contexts.

Recently, Fleischer [3] has presented an algorithm with an improved 2-approximation guarantee for the minimum-cost $\{0,1,2\}$-Survivable Network Design Problem, by adapting a technique of Jain [7]. She also describes the limitation of this LP rounding technique for solving the $k$-VC problem by exhibiting a family of examples where the largest fractional values are very small $\left(\frac{1}{k}\right)$.

Even more recently. Cheriyan, Vempala and Vetta [2] have presented a $6 H(k)$-approximation algorithm for $k$-VC for graphs with at least $6 k^{2}$ vertices, by combining some results on critically connected graphs by Mader [9] with a minimum-cost $k$-outconnected subdigraph algorithm of Frank and Tardos [4].

\section{Acknowledgments}

We thank Zeev Nutov for bringing the error reported here to our attention, and Joseph Cheriyan, Guy Kortsarz, and Tibor Jordan for discussions and comments related to it.

\section{References}

[1] Ajit Agrawal, Philip Klein, and R. Ravi. When trees collide: An approximation algorithm for the generalized Steiner problem on networks. SIAM Journal on Computing, 24:440-456, 1995 .

[2] Joseph Cheriyan, Santosh Vempala, and Adrian Vetta. An approximation algorithm for the minimum-cost $k$-vertex connected subgraph. Submitted for journal publication, 2001.

[3] Lisa Fleischer. A 2-approximation for minimum cost $\{0,1,2\}$ vertex connectivity. In Karen Aardal and Bert Gerards, editors, Proceedings of the 8th International Conference on Integer 
Programming and Combinatorial Optimization, number 2081 in Lecture Notes in Computer Science, pages 115-129. Springer, 2001.

[4] A. Frank and E. Tardos. An application of submodular flows. Linear Algebra and its Applications, 114/115:329-348, 1989.

[5] Michel X. Goemans and David P. Williamson. A general approximation technique for constrained forest problems. SIAM Journal on Computing, 24:296-317, 1995.

[6] M.X. Goemans, A.V. Goldberg, S. Plotkin, D.B. Shmoys, É. Tardos, and D.P. Williamson. Improved approximation algorithms for network design problems. In Proceedings of the 5th Annual ACM-SIAM Symposium on Discrete Algorithms, pages 223-232, 1994.

[7] Kamal Jain. A factor 2 approximation algorithm for the generalized Steiner network problem. Combinatorica, 21:39-60, 2001.

[8] Philip Klein and R. Ravi. When cycles collapse: A general approximation technique for constrained two-connectivity problems. In Proceedings of the Third MPS Conference on Integer Programming and Combinatorial Optimization, pages 39-55, 1993. Also appears as Brown University Technical Report CS-92-30.

[9] W. Mader. Endlichkeitsäze für k-kritische graphen (german). Math. Ann., 229:143-153, 1977.

[10] R. Ravi and David P. Williamson. An approximation algorithm for minimum-cost vertexconnectivity problems. Algorithmica, 18:21-43, 1997.

[11] David P. Williamson, Michel X. Goemans, Milena Mihail, and Vijay V. Vazirani. A primaldual approximation algorithm for generalized Steiner network problems. Combinatorica, 15:435-454, 1995. 